\title{
Algorithm for Size Statistic and Clustering of Block Image Sequences
}

\author{
Li Cong ${ }^{1,}$, Zou Shaoli ${ }^{2, \text { b }}$ \\ ${ }^{1}$ No 106 Jiweit Road, School of Electrical Engineering, University of Jinan, Jinan, China \\ ${ }^{2}$ No 5 Shanda Road, A judge in Shandong Training College, Jinan, China \\ a cse_lc@ujn.edu.cn, ${ }^{\mathrm{b}}$ powerlab_lc@163.com
}

Keywords: Shot blasting machine; Programmable logic controller; Ethernet; Kingview; Monitoring

\begin{abstract}
The statistic algorithm for image processing, block clustering and block size is proposed according to the block image sequences. For complicated and regular distribution block image. Firstly, we get the binary picture, and calculate the ratio of the edge and ground and the maximum distance between the two edges by the pixel scanning method, then we can get the data sample of the size and block size distribution at the same time. Secondly, using the improved nearest neighbor algorithm[5], we get the block size classification, and the percentage of each regional size in the whole area. Computer emulate result proved that this method meets the demands of the real-time image processing better for its advantage as follows: the small calculation, the high precision. The algorithm will lay a solid foundation for the operating mode analysis and automatic control.
\end{abstract}

\section{Introduction}

There need a real-time observation of the melting or calcination dynamic process of some material, in order to take analysis or corresponding parameter control. For instance, we need immediate understanding the quantity and size of the clinker caking in the cement rotary kiln calcination process, Because the quality of cement products not only related to the changes of material sintering temperature, but also related to the quantity and size of the clinker caking, and the quantity and size of the clinker caking mainly depends on the raw material ratio and appropriate mineral composition and petrographic structure. However, these processes especially like the cement rotary kiln calcination process is a very complicated production process, it is hard to establish mathematical model, and its particle size detection problems are also difficult to solve. We can use popular soft measurement technology[1] and computer vision detection technology to take block sintering images[2], which including abundant production condition information - the ratio of the edge and ground, block distribution state, then by using the image processing technology, based on identification principle to get characteristic quantity[3-4], which play a decisive role in production condition. On the base of the accumulation of a great number of statistic data, we can establish quality prediction model, and then realize the quality optimization control of the process.

The Purpose and motivation of the statistic algorithm for the block image sequences clustering and block size distribution is obvious. On the base of the accumulation of the distribution law of the block, the author acquire the simulation image of in the furnace caking with CCD in laboratory (as shown in Fig.1), and get the algorithm to solve block size distribution after repeated research and 
argumentation. This paper based on the actual proposed the method and algorithm that get the data sample of the edge by the method of scanning with specific step, and get the ratio of the edge and ground and the data sample of the distance between them; Get the block classification and size by using the improved nearest neighbor to. Computer emulate result proved that this method meets the demands of the real-time image processing better for its advantage as follows: the small calculation, the high speed, the high precision, strong practicality and so on.

\section{Edge Extraction Algorithm Research}

We can carry on binary processing for image after choose the correct threshold value t. It is assumed that the input image is $M(i, j)$, the output image is $Y(i, j)$, then: $Y(i, j)= \begin{cases}1 & M(i, j) \geq t \\ 0 & M(i, j)<t\end{cases}$

We need to extract edge contour after got the binary image.

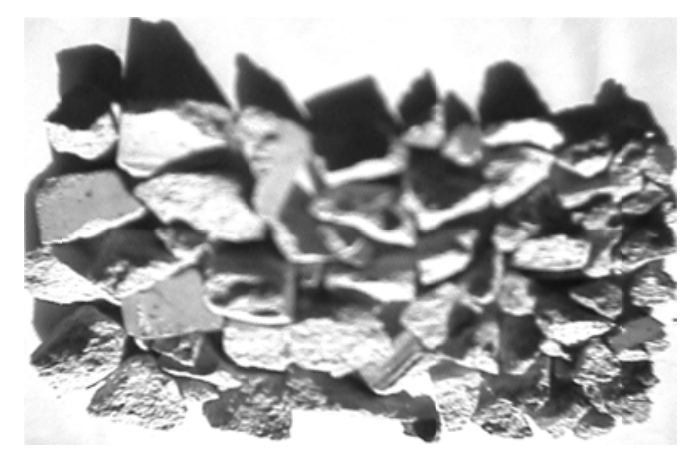

Fig.1. Simulation caking image

There are many edge detection methods, such as energy concentration filtering method, statistical edge detection method, relaxation-labeling, surface fitting operator, Marr operator, and different kinds of differential operator, etc. These methods have remarkable detection effect for one certain object; however it can't meet the demands of the on-line detection if the running time is too long. Freeman chain code is a better method to obtain size, it is a basic method to represent geometry shape of arbitrary shape in the image by discrete data.

Chain code method need edge is continuous closed curve, however, the edge of the caking is intricate, which is highly discontinuous, crossing and overlapping. It is not only time-consuming to track edge by Freeman chain code, but also easy to cause ambiguity in edge recognition so that the accuracy is lower. In the actual situation, we set the block image partition in advance in order to improve the operation speed. For the regular image we solve the classification of the caking partition from the density of pixel point that duty ratio of edge and background, and obtain the clustering situation of each area. For irregular image we consider the whole image as a partition and obtain the clustering situation by operation. The targets of them are unified.

\section{Block Clustering and Size Statistical Algorithm}

\subsection{Duty Ratio}

We get the binary images in Fig.2 (a), through the binarization of the Fig.2.(b)

We set the width of the image as $L$, the height as $H$, the imaging lattice of image as $\mathrm{M}$ (row) $\times \mathrm{N}$ (column). Firstly san the image from top to bottom, left to right in turn, calculate the total number of edge pixel points of each scanning line $P_{i}$. Through these we can get the duty ratio of 
edge and background on each scanning line as: $f\left(P_{i}\right)=p \frac{P_{i}}{N}$, the duty ratio of the area: $\frac{\sum_{i=0}^{m} p_{i}}{M^{\prime} \times N^{\prime}}$, $M^{\prime} 、 N^{\prime}$ is the number of the lattice. The function description as shown in Fig. 2 (b), the more scanning lines the more accurate the function description is.

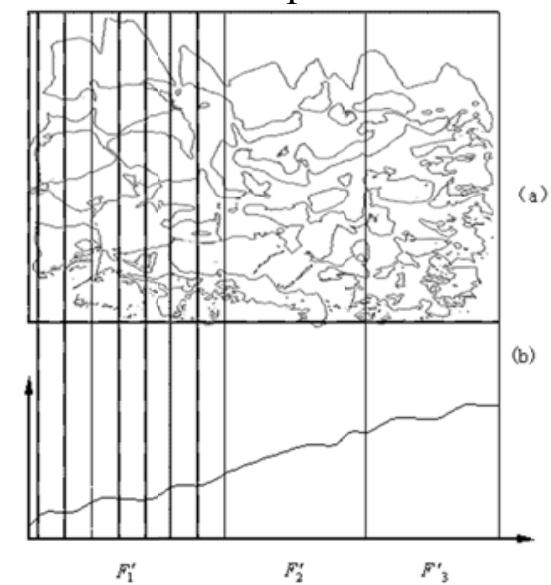

Fig.2. (a) Binary image of $\quad$ (b) Duty ratio of edge and background

\subsection{Block Size and Distribution}

Start from the origin of image, and san the image from bottom to top, left to right according to the pixel lattice distance. If found two points $y_{(j+1)}, y_{j}$ separately in the upper and lower edge on the scan line, Then we can obtain the distance between the two edges is: $s_{i j}=y_{j+1}-y_{j}, j$ is the pixel position on the $j$ th edge along $\mathrm{Y}$ axis(as shown in Fig.3).

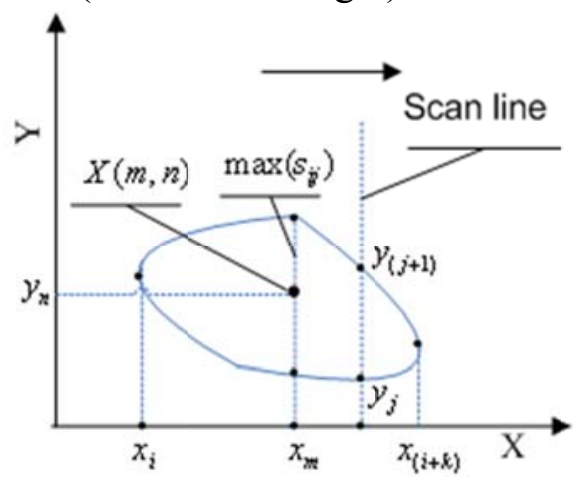

Fig.3. Schematic diagram for block size algorithm

Assuming that we calculate $s_{i j}$ value start from the pixel point $i$ on the $\mathrm{X}$ axis, we consider the block is end when $s_{(i+k) j}=0$, where $k$ is scanning step. We can get ordinate maximum size:

$\max \left(s_{i j}, s_{(i+1) j}, \cdots, s_{(i+k) j}\right)$. Recorded as $X(m, n), m, n$ is respectively size center position of $\mathrm{X}, \mathrm{Y}$ axis. Get the sample of size similarly:

$X(m, n)=\left\{X_{11}, X_{12} \ldots, X_{1 N 1}, \ldots X_{M 1}, X_{M 2}, \ldots, X_{M N_{m}}\right\}=\left\{X_{1}, X_{1} \ldots, X_{M \times\left(N_{11}+N_{2}+\cdots+N_{m}\right)}\right\}$

$N_{1}, N_{2}, \cdots . N_{m}$ represent the size number on the longitudinal.Several discussions:Decide two start position points $y_{(j+1)}, y_{j}$ of block size according to the bottom-up. $i$ is the pixel point, which is fixed value; $j$ is edge point, whose value is changing. When meet the condition that the block edge overlapped, we stop scanning and increase the number of $j$ at the same time. 
We drop the point when $\left|y_{(i+1) j_{-}} y_{i j}\right| \geq \varepsilon$ ( $\varepsilon$ is threshold value) or $y_{(i+1) j}$ is empty point.

Also can again with the $\mathrm{Y}$ axis scanned, for accurate, if time allows, can two axis scanned, will get size of combination. We can also scanning by $\mathrm{Y}$ axis, if time allows, also can scanning by two axis in order to be accurate, and combined the obtained size.

\subsection{Block Clustering}

Literature [5] demonstrated many advantages of the new clustering algorithm in detail compared to other modes clustering algorithm in detail. This paper improved on the basis of this algorithm.

Firstly, we determine a few reasonable rules to be followed before a large number of sample data is clustered into a limited clusters: Set clustering center at the area where the data is more dense; data within a neighborhood of the clustering center is categorized as one cluster, called the hinterland of this clustering center; The closeness degree between clustering center and the data within its hinterland reflect the quality of clustering results, the closer between clustering center and the data within its hinterland, the better the quality of clustering results are; The treatment process of calculate the clustering center and the classification repeatedly can make the clustering results optimized gradually.

We use the generalized distance to indicate the degree of closeness between the data, which can be defined with any norm, here we choose Euclidean norm, and the distance is:

$$
d(\mathbf{x})=d\left(\sqrt{x_{1}{ }^{2}+x_{2}{ }^{2}+\cdots+x_{n}{ }^{2}}\right)
$$

Sample $\mathbf{X}=\left(x_{1}, x_{2}, \cdots x_{n}\right)^{T}$, each sample data contains $n$ components.Now the clustering center is:

$$
\mathbf{c}_{m}=\frac{1}{J_{m}} \sum_{j=1}^{J_{m}} \mathbf{x}_{j}^{(m)}
$$

Where $\mathbf{x}_{j}^{(m)}$ is all sample in the class which clustered with $\mathbf{c}_{m}$ as the center, $j=1,2, \cdots, J_{m}, J_{m}$ is the sample number of the $m$ th clustering. $m$ used to count each clustering, Assuming there are a total of $k$ clusters, $1 \leq m \leq k$.

Separately calculated the distance between sample $\mathrm{x}$ and all clustering centers

$d\left(C_{1}, X\right) \ldots d\left(C_{m}, X\right) \ldots d\left(C_{\kappa}, X\right)$. if $d\left(C_{p}, X\right)=$ $\min _{k \rightarrow \infty}\left\{d\left(C_{1}, X\right) \ldots d\left(C_{m}, X\right) \ldots d\left(C_{k}, X\right)\right\}$

Where $1 \leq p \leq k$. Then $\mathrm{x}$ belongs to clustering $\mathbf{c}_{p}$. We rule all sample belong to clustering $\mathbf{c}_{m}$ constitute the hinterland of clustering $\mathbf{c}_{m}$, denoted by. $I C_{m}$. Assume the total number of sample as $\mathbf{E}$, the sample number the $m$ th cluster as $\mathbf{E}_{m}$, so that the rate is defined as follows: $\eta_{m}=\mathrm{E}_{\mathrm{m}} / \mathrm{E}$

The total rate is defined as follows:

$$
\eta=\sum_{m=1}^{k} \eta_{m}
$$

For the cluster centered on $\mathbf{c}_{m}$, Define its devious $D(m)$ as follows: $D(m)=\sum_{X \in I C_{m}} d\left(C_{m}, \mathrm{X}\right)$

The devious $D(m)$ reflects the devious degree of the $m$ th clustering data, or in other words 
compact degree. The total devious $D$ is defined as follows:

$$
D=\sum_{j=1}^{k} D(j)
$$

The smaller the total devious $\mathrm{D}$ is, the more compact the cluster is, and thus the better the quality of clustering result is. The clustering process: Firstly, select the points distance minimum between each other as the clustering center, and classified the samples within its certain range into its hinterland. Then choose the points distance minimum between each other as the clustering center from residual samples, and determine its hinterland. Samples have already been processed will not be considered when continue to choose clustering center and the classification. The points have a larger distance between each other will not get together. Repeat the above process until all samples are clustered. This clustering method can avoid the cluster focus on local area, The samples have a larger distance between each other will be forced to get together when the number of cluster is limited, which make the total devious very large, so that largely avoided the cluster fall into local solution. We need to make adjustments to further reduce the total devious after the initial clustering is finished, and maximize the total rate. Firstly, calculate the center of each cluster, secondly, calculate the distance between all samples and the center of each cluster and reclassified all samples according to the principle of formula (3). Adjust the clustering repeatedly until the total devious no longer decreased significantly and the total rate is maximum.

\section{The experimental results}

We get a image sequence by shooting continuously in a certain time interval. Process each picture in this sequence respectively according to the above. We conducted field simulation in the laboratory. Material stones replaced by standard concrete experiment stones, each distribution rate of the stones whose size is around the $50 \mathrm{~mm}, 35 \mathrm{~mm}$ and $20 \mathrm{~mm}$ is $20 \%, 30 \%$ and $50 \%$. We change the position of different size block properly.the adopted 20 picture constitute a set of image sequence. The extraction results of the size in 20 pictures are basically similar. We classify the stones into three clusters (can also divide them into several kinds, or take treat accordingly treatment after segmenting the images are segmented when there are longitudinal and transverse block partition at the same time). The statistical result is that stones size is $42.3 \mathrm{~mm}, 37 \mathrm{~mm}$ and $16 \mathrm{~mm}$ respectively. The rate of the major medium and small stones in the whole area is that: $50 \mathrm{~mm}$ $(40-55 \mathrm{~mm})$ caking quantity is $24.3 \%, 35 \mathrm{~mm}(25-40 \mathrm{~mm})$ caking quantity is $26.2 \%, 20 \mathrm{~mm}$ $(15-25 \mathrm{~mm})$ caking quantity accounts for $45.1 \%$. The results are satisfactory compared with the actual results. Other depth algorithm needs further improvement.

\section{References}

[1] CAO Peng-Fei, LUO Xiong-Lin. Wiener Structure Based Modeling and Identifying of Soft Sensor Systems[J]. Acta Automatica Sinica. 2014,40(10):2179-2192.

[2] Wu Xiao-xun. A Study on Burning State Recognition of Clinker Based on Burning Zone Image[D]. Shen yang: North eastern University June 2014.

[3] LIU Jin-Xin, ZHU Yun-Long, SHEN Zhe, et al. Hybrid Recognition Method for Burning Zone Condition of Rotary Kiln[J]. Acta Automatica Sinica. 2012,38(7):1153-1161.

[4] SHI Pei-yuan, DENG Tingquan. Fuzzy-based color recognition and its application in image retrieval. Computer Engineering and Applications[J], 2013, 49(18):138-141.

[5] Li Cong, Zhang Yong, Gao Zhi. A new clustering algorithm [J]. Pattem Recognition and Aitificial Intelligence. 1999,12(2):205-208. 